\title{
Analysis of Dispersion Compensation using Fiber Bragg Grating in Optical Fiber Communication System
}

\author{
Mehtab Singh \\ Assistant Professor \\ ECE Department \\ Satyam Institute of Engineering and Technology, \\ Punjab, India
}

\author{
Rajveer B. \\ Assistant Professor \\ ECE Department \\ Satyam Institute of Engineering and Technology, \\ Punjab, India
}

\begin{abstract}
Dispersion is the most important factor which determines the data rate and the maximum repeater distance spacing in a fiber optical link. In this paper, investigation has been done on performance of Fiber Bragg Grating as a technique to compensate chromatic dispersion in a $100 \mathrm{~km}$ long fiber optic link using different modulation formats such as RZ and NRZ modulation formats and different values of transmission power levels. It was observed that $\mathrm{RZ}$ modulation format showed a better performance with a maximum value of Qfactor of 54.5192 than NRZ modulation format which obtained maximum Q-factor value of 31.4792.
\end{abstract}

\section{General Term}

Performance Analysis, Dispersion compensation, Fiber Bragg Grating

\section{Keywords}

Fiber Bragg Grating; RZ; NRZ; Q- factor; power levels.

\section{INTRODUCTION}

In recent times, the use of single mode fiber (SMF) in order to transmit data at high bit rates in transmission windows having low loss has seen great importance [1], [3]. But dispersion is a matter of great concern which degrades the overall performance of fiber optic communication link. As the bit rate is increased dispersion cause the pulse to broaden at the output of the optical fiber. At high data rate, these broaden pulses may overlap with each other causing crosstalk and inter symbolic interference (ISI) which causes errors during reception of the signal at the receiver side of optical link [7]. By the use of Erbium Doped Fiber Amplifier (EDFA) in 1550 $\mathrm{nm}$ wavelength window in order to increase the distance of fiber optic link which has been limited by loss caused due to dispersion. But EDFA induces nonlinear effects in the optical fiber which limits the data rate of fiber optical channel and also reduces the maximum repeater distance spacing in fiber optic link. The combined effect of chromatic dispersion and nonlinear effect are very disastrous for data transmission in a very high data rate optical fiber link which induces overall loss in optical data transmission system [3]. Hence, some dispersion management techniques must be used in order to suppress impairments caused by chromatic dispersion and non linear effects so as to achieve high data rates in the optical fiber link. Several techniques have been proposed which can be used to overcome power penalty caused due to non linear effects and chromatic dispersion. These include differential delay methods [4], initial pre-chirp [5], dispersion compensating devices [6], mid spam spectral inversion [7-9], dispersion supported transmission [10-13], optical phase conjugation [14-18]. The use of dispersion compensated fiber (DCF) is considered as the best method to mitigate harmful results of dispersion and also to upgrade single mode fiber links which had already been installed. Dispersion compensation fiber has a high value of negative dispersion which is used to compensate for positive dispersion caused due single mode fiber. For long distance communication link, we use the combination of SMF along side with dispersion compensated fiber in order to compensate dispersion. Three schemes of dispersion compensation can be used in DCF compensation scheme (pre, post or symmetrical compensation) [1]. Electronic dispersion compensation (EDC) is another technique which can be used to compensate dispersion by using feed forward equalization or decision feedback equalization or both to compensate dispersion. Another method of dispersion compensation is use of Fiber Bragg Grating in order to compensate dispersion caused due to optical fiber link. In Fiber Bragg Grating different wavelength are reflected inside of grating at different distance [2]. The larger wavelength travels a larger distance before reflection and the shorter wavelength travels a comparatively lesser distance inside the fiber. As a results the pulse which was expanded by dispersion has now been compressed by use of Fiber Bragg Grating [19].

In this paper discusses use of FBG to compensate dispersion in a $100 \mathrm{~km}$ long fiber optical link. Different modulation formats such as RZ and NRZ modulation formats are used at transmitter side and their results are compared. Different transmission power levels are used to transmit data and their results are compared. The value of Q-factor, eye height and bit error rate (BER) is determined for each and every case and their values are compared. Fiber Bragg Grating is discussed in detail in section 2. In section 3 simulation setup for performance analysis is shown. Results and discussion are presented in section 4 . In section 5, concluding remarks are given.

\section{FIBER BRAGG GRATING (FBG)}

The concept of Fiber Bragg Grating was first introduced in 1980 and has been used in several applications and widely researched. It consists of a linear reflective device whose reflective index profile changes linearly with respect to length of the fiber. The grating reflects the light depending upon the wavelength of the light entered in the grating [20]. The light with larger wavelength travels a greater distance in the grating before getting reflected on the other hand ray with smaller wavelength travels a shorter distance inside the grating before getting reflected. As a result, the ray which is expanded by the 
chromatic dispersion in an SMF is compressed by passing through a Fiber Bragg Grating [19]. This is the basic principle of Fiber Bragg Gratings. The reflected wavelength $\left(\lambda_{b}\right)$ also known as the Bragg wavelength is given by the relationship:

$$
\lambda_{b}=2 n \Lambda
$$

Where $\mathrm{n}$ is the effective refractive index of the grating in the fiber core and $\Lambda$ is the grating period. The main advantage of using Fiber Bragg Grating as a dispersion compensation technique is that it is very cost effective and has a low insertion loss and the are passive components compatible with single mode fiber. FBG also find its application in different a field such as WDM add/drop filters, pump lasers and wavelength stabilizers [20]. Fig no. 1 shows the basic principle of FBG.

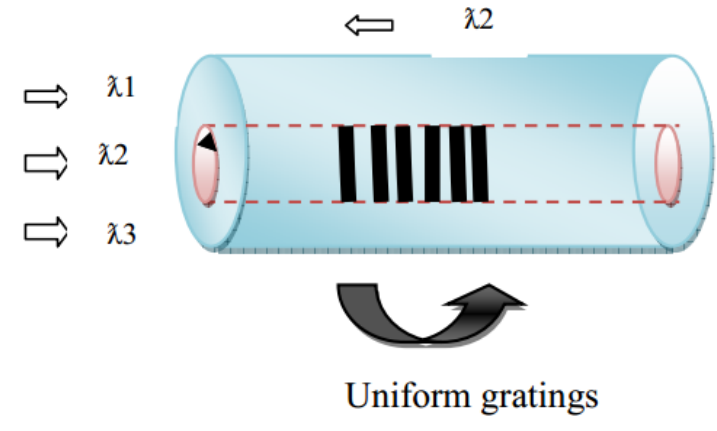

Fig no. 1 Principle of Fiber Bragg Grating [4]

The functioning of FBG depends upon the reflection of light from gratings and coupling of modes [6]. Forward and backward propagating fields of the same mode causes coupling and they show strong coupling if they satisfy following condition:

$$
\beta_{1}-\beta_{2}=2 m \pi / \Lambda
$$

where $\beta_{1}$ and $\beta_{2}$ are phase constants of two modes. Now if we consider two modes which are counter propagating we have

$$
\beta_{2}=-\beta_{1}
$$

And hence the Bragg Diffraction condition is given by:

$$
2 \beta=2 \mathrm{~m} \pi / \Lambda
$$

If effective modal index is given by $\mathrm{n}_{\mathrm{eff}}$ then:

$$
\beta_{1}=2 \pi \mathrm{n}_{\mathrm{eff}} / \lambda
$$

Thus, the Bragg wavelength which is strongly reflected by grating is given by:

$$
\lambda_{b}=2 n_{e f f} \Lambda
$$

This is the final condition of the wave to be reflected inside of a Fiber Bragg Grating.

\section{SIMULATIONS SETUP}

In this paper, simulations have been performed using two different modulation formats namely RZ and NRZ modulation formats at different transmission power levels. Various simulations parameters utilized in this analysis work are given in Table no. 1 and various fiber parameters used in this simulation is given in Table no. 2 .

Table no. 1 Simulations Parameters

\begin{tabular}{|c|c|}
\hline Parameters & Value \\
\hline Bit rate & $2.5 \mathrm{Gbps}$ \\
\hline Sequence Length & 64 \\
\hline Samples per bit & 256 \\
\hline Central frequency & $193.1 \mathrm{THz}$ \\
\hline Extinction ratio & $30 \mathrm{db}$ \\
\hline Power & $-5 \mathrm{dBm}-18 \mathrm{dBm}$ \\
\hline Bandwidth & $125 \mathrm{GHz}$ \\
\hline Dispersion FBG & $-800 \mathrm{ps} / \mathrm{nm}$ \\
\hline
\end{tabular}

Table No. 2 Fiber Parameters

\begin{tabular}{|c|c|}
\hline Parameters & SMF \\
\hline Length $(\mathrm{km})$ & 100 \\
\hline Attenuation $(\mathrm{db} / \mathrm{km})$ & 0.2 \\
\hline $\begin{array}{c}\text { Dispersion } \\
(\mathrm{ps} / \mathrm{nm} / \mathrm{km})\end{array}$ & 16 \\
\hline $\begin{array}{c}\text { Dispersion slop } \\
(\mathrm{ps} / \mathrm{nm} 2 / \mathrm{km})\end{array}$ \\
\hline
\end{tabular}


Simulation setup used in this analysis are given in Fig no. 2 and Fig no.

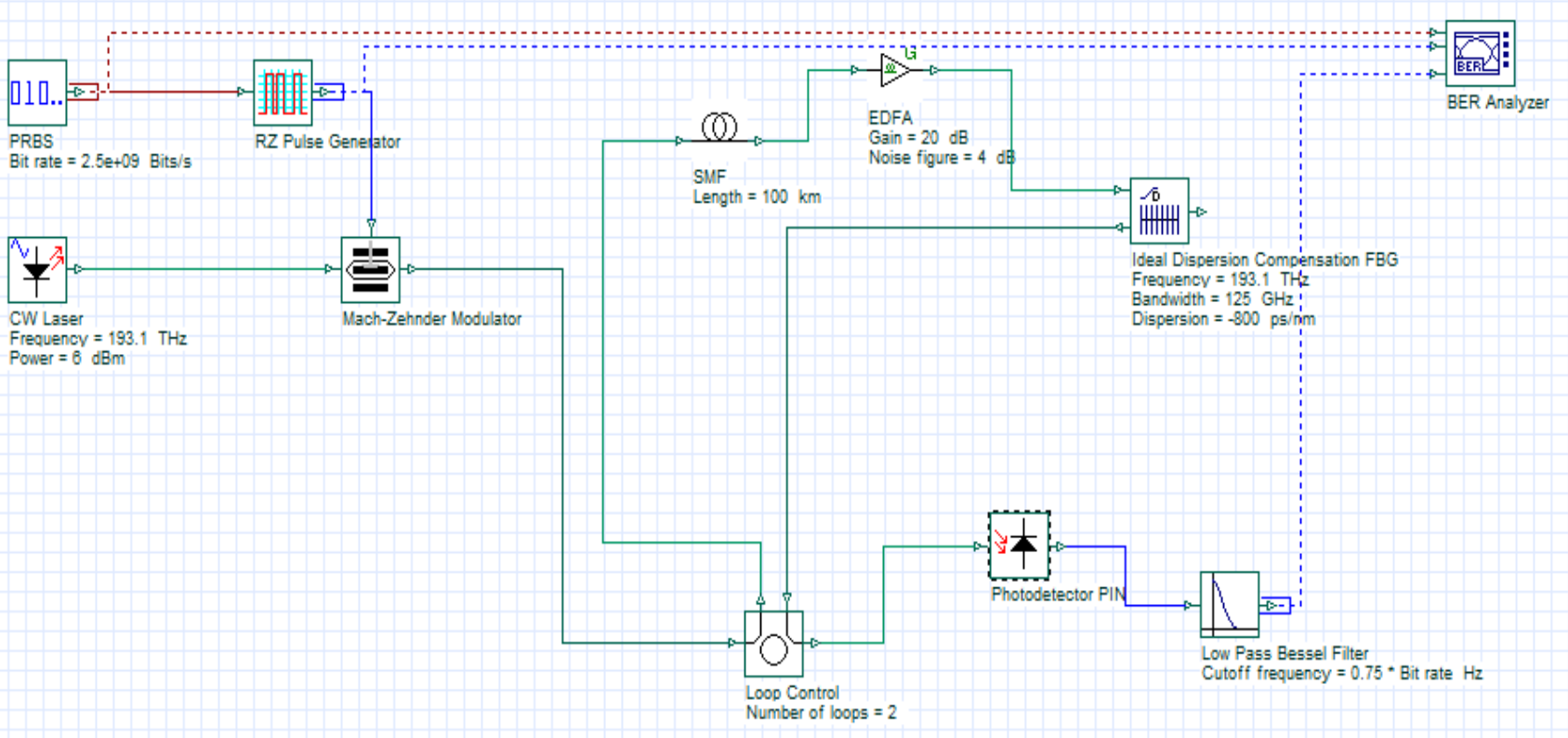

Fig no. 2 Simulation setup for RZ modulation format

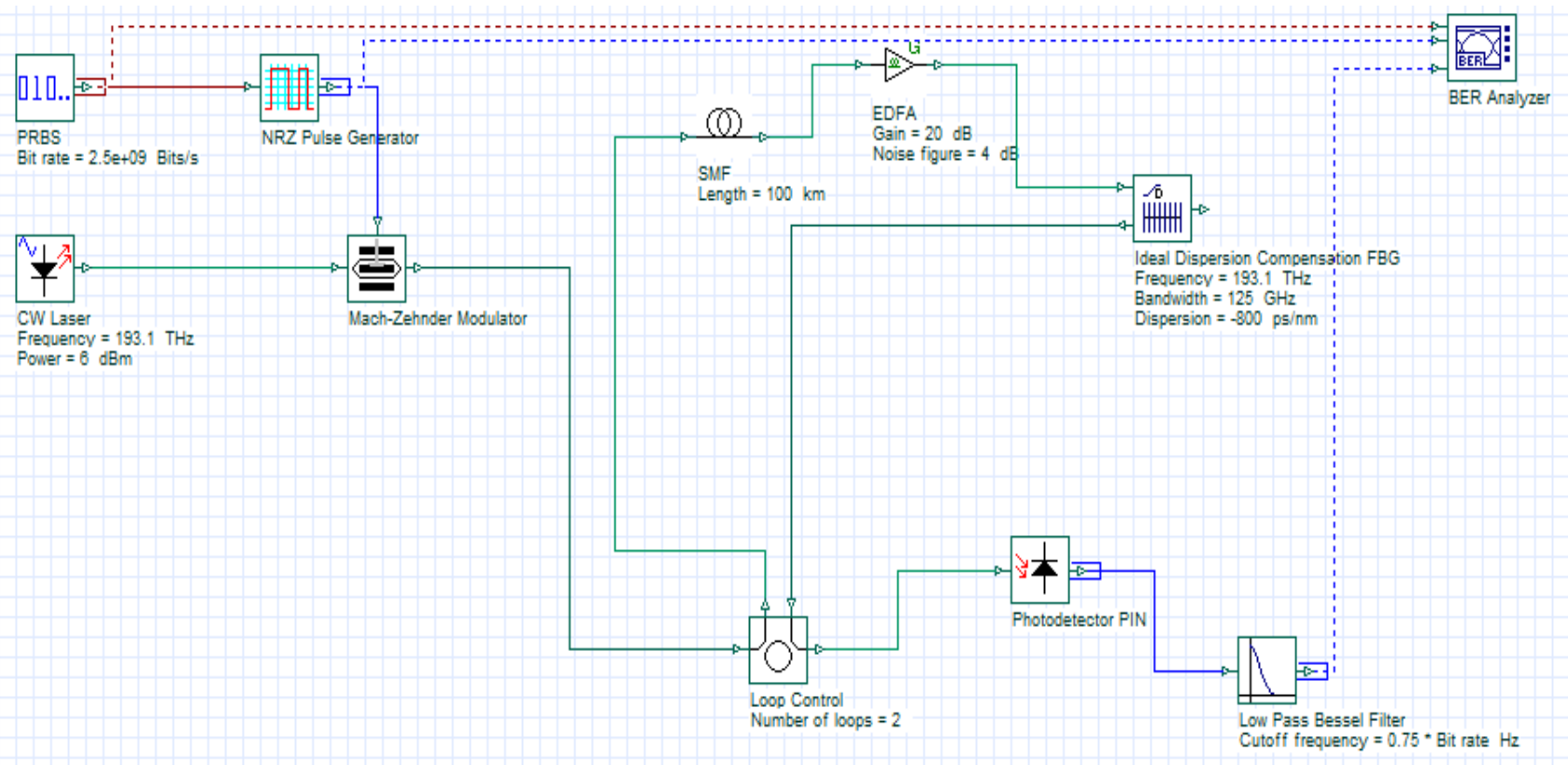

Fig no.3 Simulation setup for NRZ modulation formats

At first we have used a pseudo-random bit generator which transmits a sequence of 0 's and 1's which are fed into the input stage of either RZ or NRZ modulators. This string of 0's and 1's is converted into electrical pulses which are fed to input of Mach Zender modulator which modulates the signal with a continuous wave centered at frequency $193.1 \mathrm{THz}$. The signal is then fed tom an SMF of length $100 \mathrm{~km}$. An EDFA is used to compensate loss due to large span of single mode fiber. Then the signal is passed through the FBG for compensation. The optical information signal is then retrieved by PIN photo diode which converted optical signal to electrical signal. This signal is then passed through an LPF in order to remove all high-frequency noise. The signal is finally analyzed by BER which determines various performance parameters such as BER, Q-factor, eye height. 


\section{RESULTS AND DISCUSSIONS}

In this proposed system evaluation has been done on the performance of Fiber Bragg Grating in order to compensate dispersion depending upon the types of modulation formats used and also on the transmitted power levels. In order to carry out various simulations, Optisystem 7.0 has been used..

The eye diagram figures for RZ and NRZ modulation formats at $193.1 \mathrm{THz}$ frequency are shown in Fig No. 4 and Fig No. 5 respectively.

The comparison for values of BER, eye height, and Q- factor for RZ and NRZ modulation formats at different transmission power levels are presented in table no. 3 and table no. 4 respectively.

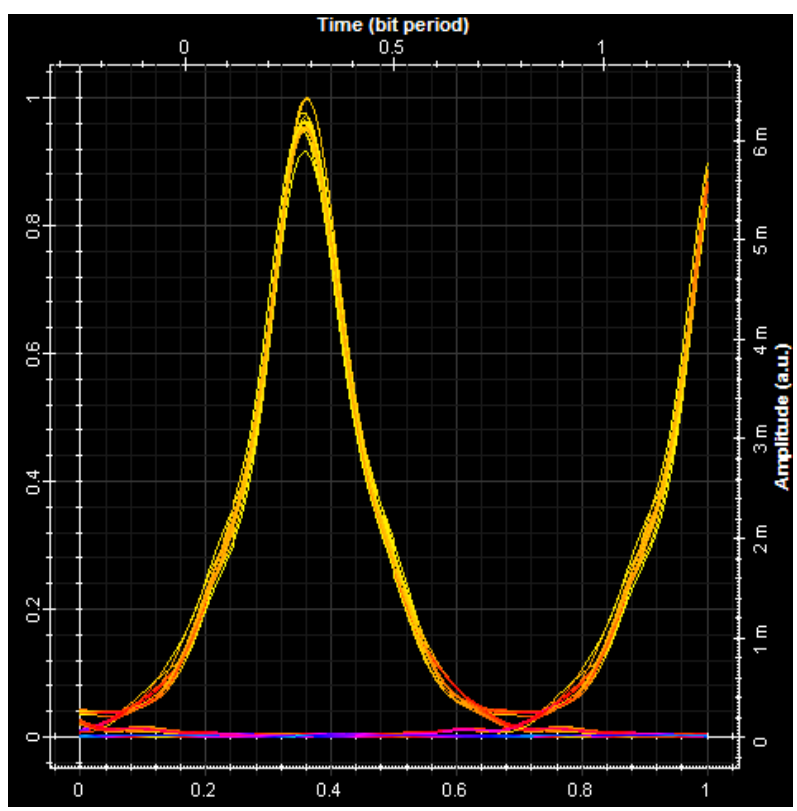

Figno.4 Eye Diagram for RZ modulation format at 193.1 THz

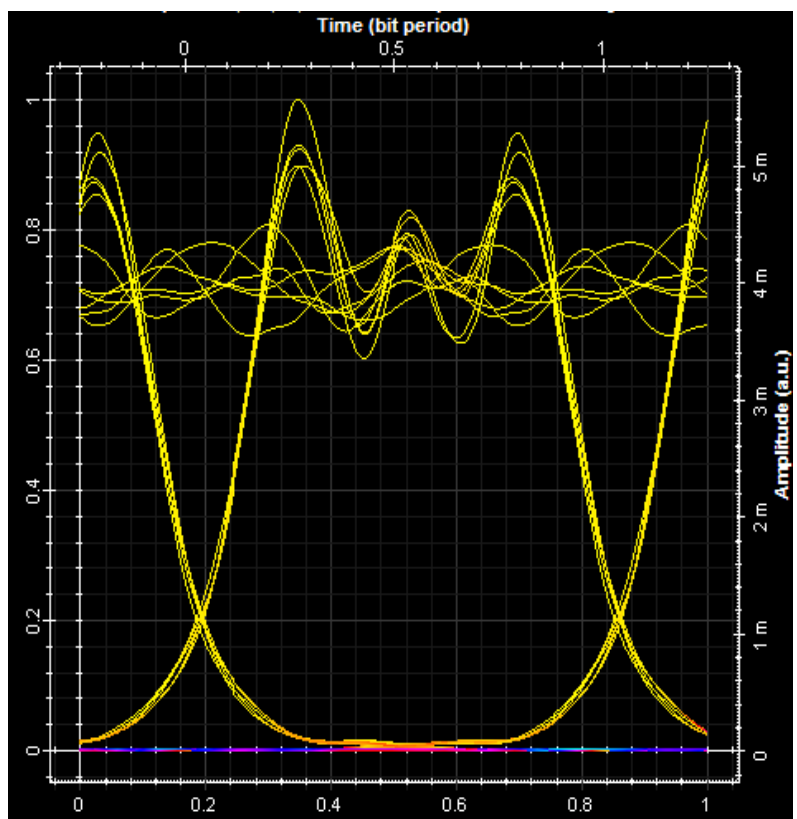

Figno.5 Eye Diagram for NRZ modulation format at 193.1THz
Table no.3 Comparison of values of Q-factor, Eye Height, and BER at different power levels for $R Z$ modulation

format

\begin{tabular}{|l|l|l|l|}
\hline $\begin{array}{l}\text { Power } \\
(\mathbf{d B m})\end{array}$ & Q- Factor & Eye Height & BER \\
\hline-5 & 22.4517 & 0.000406007 & $4.59 \mathrm{e}-112$ \\
\hline 0 & 41.923 & 0.00138175 & 0 \\
\hline 2 & 49.3289 & 0.0022266 & 0 \\
\hline 6 & 54.5192 & 0.00573776 & 0 \\
\hline 10 & & & \\
\hline 14 & 43.4614 & 0.0147851 & 0 \\
\hline 18 & & & $3.02 \mathrm{e}-165$ \\
\hline
\end{tabular}

Table no.4 Comparison of values of Q-factor, Eye Height, and BER at different power levels for NRZ modulation format

\begin{tabular}{|c|c|c|c|}
\hline $\begin{array}{c}\text { Power } \\
(\mathbf{d B m})\end{array}$ & Q-Factor & Eye Height & BER \\
\hline-5 & 23.4541 & 0.000272546 & $4.51 \mathrm{e}-122$ \\
\hline 0 & 31.4792 & 0.000908303 & $5.82 \mathrm{e}-218$ \\
\hline 2 & 30.6267 & 0.00144721 & $1.78 \mathrm{e}-206$ \\
\hline 6 & 26.1197 & 0.00362608 & $8.13 \mathrm{e}-152$ \\
\hline 10 & 21.7345 & 0.00899222 & $2.61 \mathrm{e}-105$ \\
\hline 14 & 20.5543 & 0.0216379 & $1.79 \mathrm{e}-094$ \\
\hline 18 & 10.9121 & 0.0517604 & $2.19 \mathrm{e}-028$ \\
\hline & & & \\
\hline
\end{tabular}

\section{CONCLUSION}

This informative article targets the analysis of the performance of Fiber Bragg Grating to compensate dispersion for different modulation schemes at different transmission power levels. From the above presented results, it is concluded that performance of RZ modulation scheme is better as it showed the highest value of Q-factor of 54.5192 as compared to that of NRZ which achieved a maximum value of 31.4792. As a result of above carried out analysis, it is clear 
that RZ modulation scheme gives a better performance in optical fiber communication than NRZ modulation scheme. From the above results, it can be concluded that RZ modulation scheme is a better option than NRZ modulation scheme for the purpose of transmission in optical transmission system.

\section{ACKNOWLEDGMENTS}

The authors would like to express their thanks to the Department of Electronics \& Communication Engineering, Satyam Institute of Engineering \& Technology, Amritsar, India for their support and encouragement in developing the system and for carrying out this research work in the research and development lab.

\section{REFERENCES}

[1] K.Tajima, L.M.Washio, Opt.Lett.10 (9) (1985) 460.

[2] T.L. Koch, R.C. Alferness, J. Lightwave Technol. LT3(1985) 800 .

[3] R.S. Kaler, T.S. Kamal, A.K. Sharma, S.K. Arya, R.A. Aggarwala, J.Fiber Integrated Opt.21 (3) (2002) 193.

[4] A.H. Gnauck, S.K. Korotky, J.J. Veselka, J. Nagpal, C.T. Kemmerer, W.J. Minford, D.T. Moser, IEEE, Photon. Technol.Lett.3 (10) (1991).

[5] J.J. O'Reilly, M.S. Chauldry, IEEE, Colloquim Microwave Optoelectron.139 (1990) 13/1.

[6] R.M. Jopson, A.H. Gnaules, R.M. Derosier, in: Proc. OFC'93, paper pd3, 1993.

[7] A.Yariv, D.Feke, D.M.Pepper, Opt.Lett.4 (1979) 52.
[8] S.Watanable, T.Naito, T. Chikma, IEEE, Photon. Technol.Lett.5 (1) (1993) 92.

[9] C.D. Poole, J.M. Wieserfed, D.J. DiGiovanni, A.M.Vengsarkar, J.Lightwave Technol. 12 (10) (1994) 1746. [10] R.-D. Li, P. Kumar, W.L. Kath, J. Lightwave Technol. 12 (3) (1994) 541.

[10] J.C. Cartledge, H. Debregeans, C. Rolland, IEEE, Photon. Technol.7 (2) (1995) 224.

[11] N.Henmi, T. Saito, T.Ishida, J.Lightwave Technol. 12 (10) (1994) 1706.

[12] A.J. Antos, D.K. Smith, J. Lightwave Technol. 12 (10) (1994) 1739.

[13] A.Djupsjobak a, O.Sahlen, IEEE, J. Lightwave Technol. 12 (10) (1994) 1849.

[14] A.K. Sharma, R.K. Sinha, R.A. Agarwala, J. Fiber Integrated Opt.16 (4) (1997).

[15] A.K. Sharma, R.K. Sinha, R.A. Agarwala, J. Opt. Fiber Technol.4 (1998) 135.

[16] A.K. Sharma, R.K. Sinha, R.A. Agarwala, Int. J. Light Electron Opt., Optik 111 (7) (2000) 310.

[17] G.P. Aggrawal, Fiber Optic Communication Systems, wiley, New York, 1997.

[18] C.Peuchere t, N.Hanik, R.Freund, L.Molle, P.Jeppesen, IEEE Photon.Technol.Lett.12 (8) (2000) 992.

[19] R.J. Nuyts, Y.K. Park, P. Gallison, Photon. Technol. Lett. 8 (1996) 1406. 